\title{
Management of different challenging cases of invasive cervical root resorption using biodentine: Case reports
}

\author{
H. C. Baranwal ${ }^{1}$, Aniqua Sami ${ }^{2}$, Nidhi Singh ${ }^{3^{*}}$ \\ ${ }^{1}$ Professor, ${ }^{2}$ Junior Resident, ${ }^{3}$ Senior Resident, ${ }^{1-3}$ Dept. of Conservative dentistry and Endodontics, ${ }^{1-3}$ Faculty of Dental Sciences, IMS, \\ BHU Varanasi, Uttar Pradesh, India
}

Corresponding Author: Nidhi Singh

Email: drnidhiconsendo@gmail.com

\begin{abstract}
Invasive cervical resorption is relatively uncommon and proceeds in a gradual and often aggressive destructive form of external root resorption. Etiology of invasive cervical resorption is not very clear. It is sometimes misdiagnosed which leads to improper management of effected tooth or tooth loss. Only accurate diagnosis and proper management can lead to successful outcome. The goal of treatment to suppress all resorbing tissues and the reconstruction of resorptive defect by the placement of a suitable filling material or some biological systems. Resorption area identified, cleaned \& cautery with $90 \%$ tri chloroacetic acid, further with saline. Repaired with biodentine and sutured. The aim of this case report was to reveal the management of invasive cervical resorption by using biodentine.
\end{abstract}

Keywords: Invasive cervical resorption, External root resorption, Trichloracetic acid, Biodentine.

\section{Introduction}

Invasive cervical resorption is defined as a lytic resorptive process which involves cementum or cementum and dentin of the roots of teeth, involves the surface of root below epithelial attachment and coronal aspect of the supporting alveolar process, namely the zone of connective tissue attachment ${ }^{1}$. It is an ominous and often aggressive form of external root resorption. Pulp has no role in the root resorption and it can involve any permanent tooth.

Because of its destructive resorptive property there is loss of tooth structure. The affected tooth may show pinkish discolouration of tooth crown because of resorption of coronal dentin and enamel that has highly vascular resorptive tissue.

Invasive cervical root resorption starts just apical to the epithelial attachment, most commonly it is seen in cervical area but it may be seen anywhere in the root. ${ }^{2}$ In the initial stage it may be symmetrical but in advance stage it is asymmetrical. $^{3}$

Source of invasive cervical root resorption is although unknown, the suspected cause are trauma and stimulate by sulcular microorganisms which leads to inflammation in periradicular tissues. ${ }^{4-6}$ Dentoalveolar and orthognathic surgery, orthodontic tooth movement, granuloma, cyst, central jaw tumors, replantation of teeth, impaction of tooth, periodontal treatment, bleaching and systematic diseases are predisposing factors. ${ }^{4,7-10}$

Radiographically, it appears as ragged area or scooped out area on the root surfaces or as blunting of the apex. The lesions may have well-defined radiolucencies with irregular borders and it may sometimes appear as caries radiographically.

There are various materials which have been introduced to seal the resorptive defect such as glass-ionomer cement (GIC), mineral trioxide aggregate (MTA), calcium enriched mixture (CEM) etc. MTA has been proved to be biocompatible and also acceptable in vivo biological performance when used for filling root canals with mature and open apices of teeth, perforation repair, pulp capping, pulpotomy and apexification treatment. More recently, other bioactive materials, such as CEM cement, bioaggregate, biodentine, endosequence root repair material (ERRM), and endosequence BC sealer are also used. ${ }^{11}$

Among the options available, a new bioactive cement, Biodentine (Septodont, St. Maur-des-Fosses, France) is a suitable material for repair of cervical resorption because mechanical properties of biodentine is similar to that of dentine. When it was used to seal perforations in the furcal area, it induced the repair of the periodotium and new cementum formation over the material. ${ }^{12,13}$

Therefore, the present case reports were about describing the management of type-IV Heithersay ${ }^{10}$ clinical classification of invasive cervical resorption surgically by using Biodentin.

\section{Case Report 1}

A 24 year old male patient came to the department of conservative dentistry and endodontics, with the chief complaint of mobility of bridge in upper right and left front tooth region since 3-4 months [Fig. 1a]. Patient gave past dental history that he had undergone trauma 8 years back with root canal treatment in 13,12 and 21 , further bridge in relation to 12,1121 . On clinical examination there was mobility in the bridge. On radiographic examination there was severe invasive cervical resorption in relation to 12 and 21 [Fig. 1b]. In which there was total crown root in relation to 12 . So, 12 was extacted after bridge removal. Initially gutta percha was removed then canal was cleaned and irrigated with $2.5 \%$ hypocloride and EDTA, then obturated below the resorption area [Fig. 1c]. Under LA a full mucoperiosteal flap was elevated [Fig.1d], resorption area was identified, cleaned and cautery with $90 \%$ trichloroacetic acid, further with saline, repaired with biodentine [Fig.1e] and then sutured. Recalled the patient after one month [Fig. 1f] and next after eleven months [Fig.1g] 


\section{Case Report 2}

A 26 years old female patient came to the department of conservative dentistry and endodontics with the chief complaint of swelling in upper right front tooth region since 10-15 days. Patient gave history of trauma 6 years back and tooth was untreated. On clinical examination there was percussion to tender and swelling was present in relation to 13 , at its labial aspect with slight discoloration which increases progressively. On radiographic examination there was severe invasive resorption in relation to 13 [Fig. 2a]. The electric pulp test was negative. So, the diagnosis was irreversible pulpitis with external ICR.

A surgical repair of perforation of invasive cervical resorption by Biodentine was planned after access the canal followed by CMP and obturation by gutta percha and Bioroot RCS (septodont) which is biodentine based root canal sealer [Fig. 2b,2c]. Recalled the patient after three months. [Fig. 2d] and twelve months [Fig. 2e] for follow-up

\section{Discussion}

Successful outcome in management of each case of invasive cervical resorption, must be related to the etiology, a radiographic examination is essential to the diagnosis of invasive cervical resorption and it is difficult to differentiated from internal root resorption. CBCT is a very useful diagnostic tool for correct diagnosis of invasive cervical resorption and internal root resorption. Trauma was assumed one of the predisposing factors which cause the development of invasive cervical resorption in the both present case.

There are different modalities for the treatment of invasive cervical root resorption which have been suggested by several authors. A successful outcome for such cases generally involves early diagnosis, elimination of the resorption, and restorative management. When invasive cervical resorption is diagnosed, three choices are generally considerable for treatment:

1. No treatment with eventual extraction when the tooth becomes symptomatic;

2. Immediate extraction; or

3. Access, debridement, and restoration of the resorptive defect. $^{14}$

The main aim for the management of invasive cervical resorption is the complete removal of the resorptive tissue and the restoration of the defected area. Debridement of resorption lesion is done by chemical escharotic agents such as trichloroacetic acid (TCA) which completely eliminate the resorbing cells and penetrate into the defect and increases the visualization of defect. 90\% TCA causes coagulation necrosis of granulation tissue, making it avascular, thus fascilitating the removal. TCA also has the potential to deactivate the tissue present in infiltration channels and recesses. ${ }^{15}$ Management of the resorption and the root canal treatment was done in single appointment to prevent any secondary infection. ${ }^{16}$

Like MTA, Biodentin has also been proved as a favorable repair material because of its biocompatibility and bioactivity. Biodentin is a calcium silicate based material use for repair of perforations and resorption, apexification and root-end fillings. It can also be use in class-II fillings as a temporary enamel substitute and in large carious lesions as a substitute of permanent dentin. Biodentin preservs the pulp vitality and promotes its healing process. So, it is also used in indirect and direct pulp capping and pulpotomy. The powder of biodentin contains-tricalcium silicate, dicalcium silicate, calcium carbonate, zirconium dioxide. The liquid contains calcium chloride in aqueous solution, with an admixture of polycarboxylate. Biodentine has shorter sitting time that is less than $12 \mathrm{~min}$ and high mechanical properties with excellent sealing ability. It has property to release calcium ion and inhancing the alkaline environment, which make biodentin more conductive for osteoblastic activity. ${ }^{12,13}$ Calcium and hydroxide ions also stimulate the release of alkaline phosphatase, pyrophosphatase and bone morphogenetic protein-2, which favors the mineralization process. ${ }^{17}$ Interestingly, biodentin produce the greatest quantity of PDL cells $(p<0.05)$. Biodentin and MTA ensue a significantly higher concentration of osteoblast and PDL cells. The remaining materials disclose a lower density of PDL cell and osteoblasts. It has been also proved that on human gingival fibroblasts and MG63 osteoblast-like cells, biodentin is non-cytotoxic and non genotoxic. ${ }^{18}$ It also did not affect the proliferation of pseudo-odontoblastic cells (MDPC-23) which was studied in a three dimensional multicellular spheroid model and in the presence of biodentin the expression of levels of collagen was significantly higher.

These cases show that invasive cervical resorption can be arrested using the Heithersay approach for treatment that is mechanical debridement with TCA and restorations. Present case selection and proper negotiation can lead to the successful treatment and long term retention of the tooth. Although these case reports present a favorable outcome, further studies are encouraged to support the use of biodentin to fill external invasive cervical resorption. 


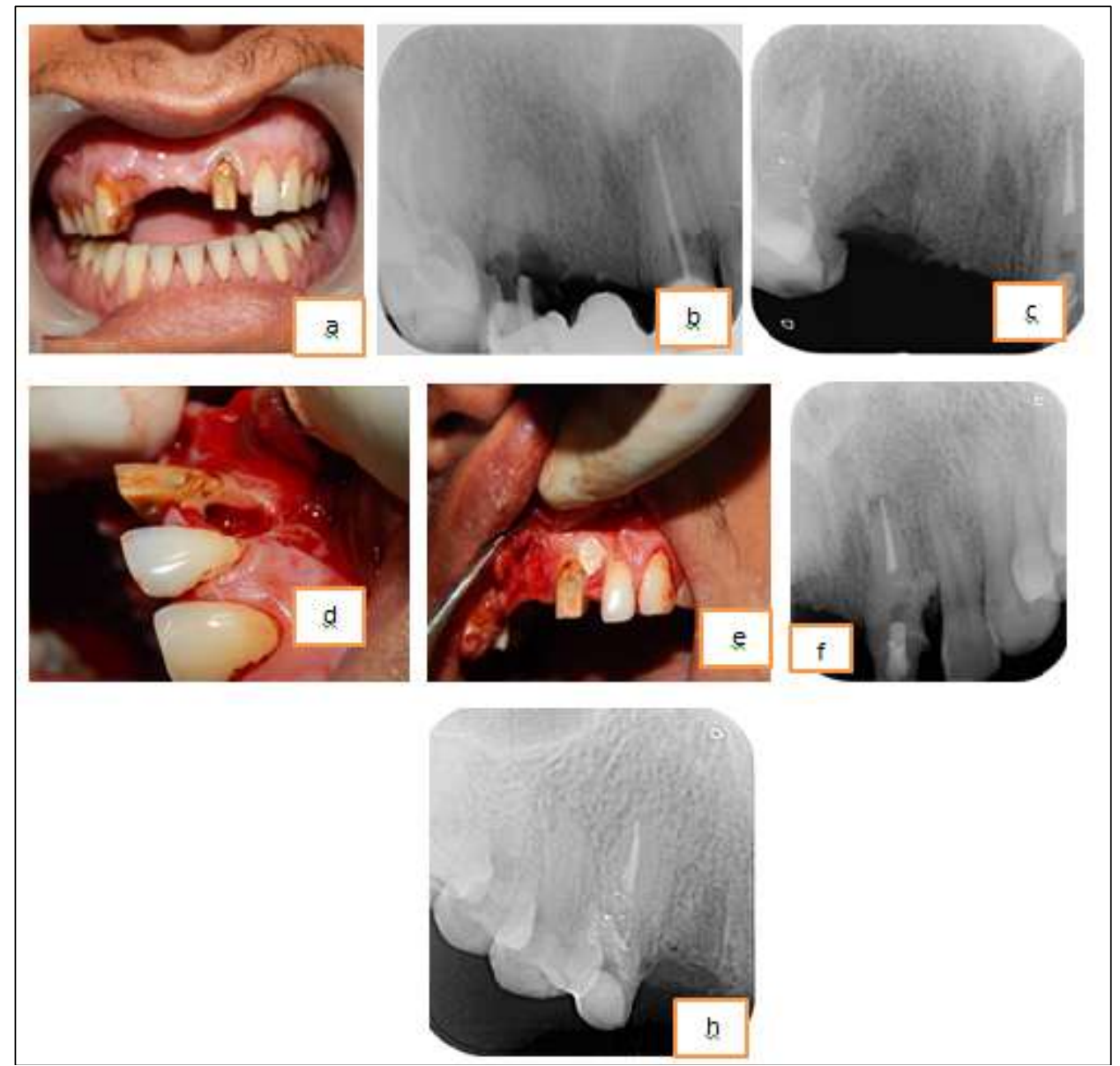

Fig. 1: (a) Pre-operative clinical photograph; (b) Pre-operative radiograph; (c) IOPA radiograph after 12 was extracted; (d) Cervical resorption defect; (e) Sealing with biodentine; (f) After one months follow-up; (g) After eleven months follow-up

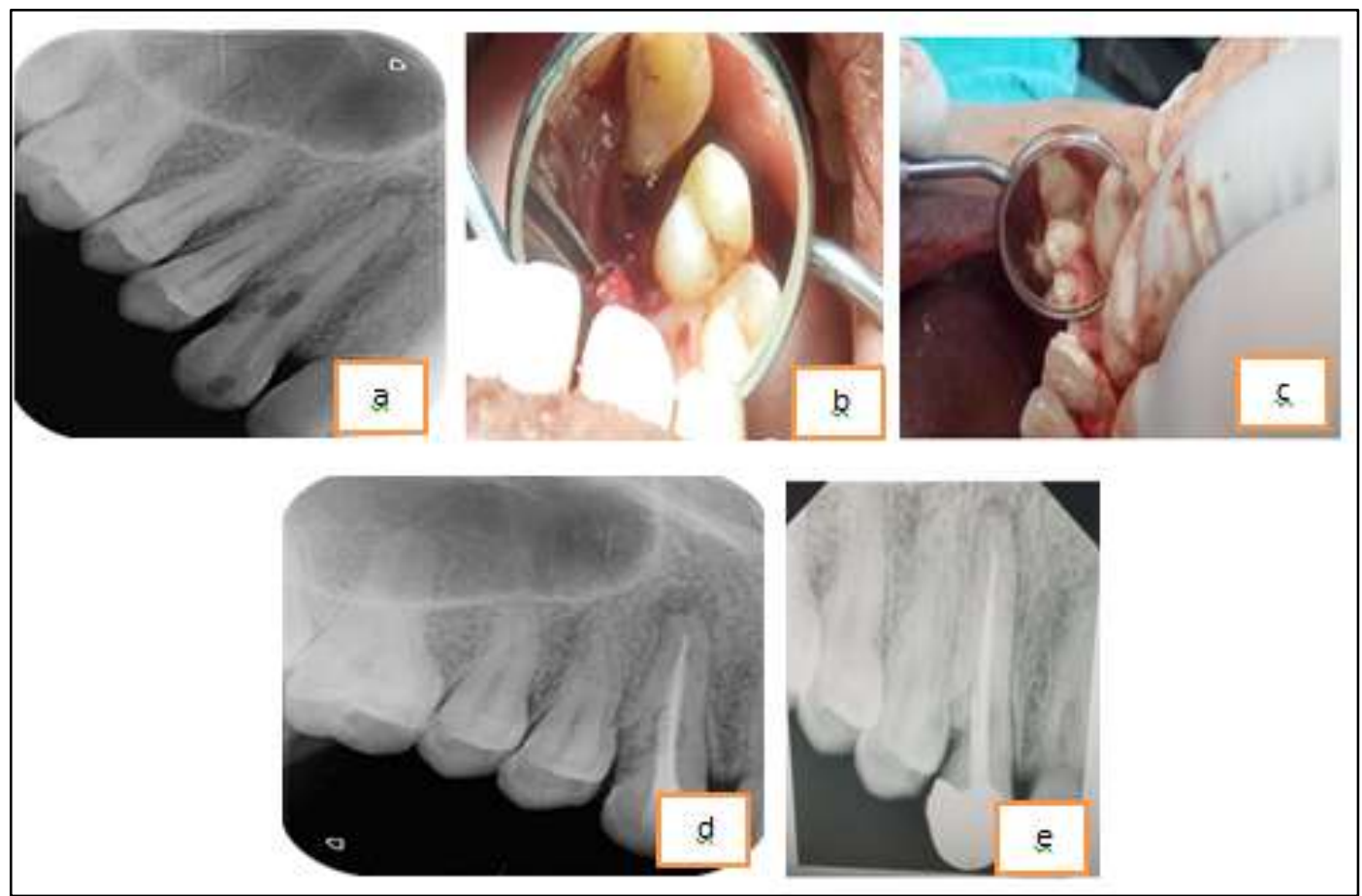

Fig. 2:(a) Pre-operative radiograph; (b) After granulation tissue removal; (c) Repaired with biodentine; (d) Follow-up after three months; (e) After twelve months follow-up 


\section{Conclusion}

Managements of these type of cases are always challenging. With proper treatment and use of modern endodontic techniques and Bioceramic materials, the prognosis of extensively resorbed or even perforated cases is fairly good.

\section{Conflict of Interest: None.}

\section{References}

1. Tronstad L. Root resorption--etiology, terminology and clinical manifestations. Endod Dent Traumatol 1988;4:241-52. Back to cited text no. 2 [PUBMED].

2. L. Bergmans, J. van Cleynenbreugel, E. Verbeken, M. Wevers, B. van Meerbeek, and P. Lambrechts, "Cervical external root resorption in vital teeth: X-ray microfocus-tomographical and histopathological case study," J Clin Periodontol 2002;29(6):580-5.

3. S. Patel, S. Kanagasingam, and T. P. Ford, "External cervical resorption: a review," J Endod 2009;35(5):616-25.

4. L. Tronstad, "Root resorption - etiology, terminology and clinical manifestations," Endod Dental Traumatol 1988;4(6):241-52.

5. M. Trope, "Root resorption of dental and traumatic origin: classification based on etiology," Pract Periodontics Aesthet Dent 1998;10(4):515-22.

6. S. I. Gold and G. Hasselgren, "Peripheral inflammatory root resorption. A review of the literature with case reports," J Clin Periodontol 1992;19(8):523-34.

7. Harrington GW and Natkin E, "External resorption associated with bleaching of pulpless teeth," J Endod 1979;5(11):344-8.

8. Madison S and Walton R, "Cervical root resorption following bleaching of endodontically treated teeth," J Endod 1990;16(12):570-74.

9. Frank AL and Torabinejad M, "Diagnosis and treatment of extracanal invasive resorption," J Endod 1998;24(7):500-04.
10. Heithersay GS, "Invasive cervical resorption: an analysis of potential predisposing factors," Quintessence Int 1999;30(2):83-95.

11. Utneja S, Nawal RR, Talwar S, Verma M. Current perspectives of bio-ceramic technology in endodontics: Calcium enriched mixture cement - Review of its composition, properties and applications. Restor Dent Endod 2015;40:1-13.

12. Malkondu Ö, Karapinar Kazandağ M, Kazazoğlu E. A review on Biodentine, a contemporary dentine replacement and repair material. Biomed Res Int 2014;2014:160951.

13. Priyalakshmi S, Ranjan M. Review on Biodentine-A bioactive dentin substitute. J Dent Med Sci 2014;13:13-7.

14. Schwartz RS, Robbins JW, Rindler E. Management of invasive cervical resorption: Observations from three private practices and a report of three cases. $J$ Endod 2010;36:1721-30.

15. Heithersay GS. Treatment of invasive cervical resorption: An analysis of results using topical application of trichloracetic acid, curettage, and restoration. Quintessence Int 1999;30:96110

16. G. S. Heithersay, "Clinical, radiologic, and histopathologic features of invasive cervical resorption," Quintessence Int 1999;30(1):27-37.

17. Gandolfi MG, Siboni F, Polimeni A, Bossù M, Riccitiello F, Rengo $\mathrm{S}$ et al. In vitro screening of the apatite-forming ability, biointerctivity and physical properties of a tricalcium silicate material for endodontics and restorative dentistry. Dent $J$ 2013;1:41-60.

18. Perard M, Le Clerc J, Meary F, Perez F, Tricot-Doleux S, Pellen-Mussi P. Spheroid model study comparing the biocompatibility of Biodentine and MTA. J Mater Sci Mater Med 2013; 24(6):1527-34.

How to cite this article: Baranwal HC, Sami A, Singh N. Management of different challenging cases of invasive cervical root resorption using biodentine: Case reports. Ann Prosthodont Restor Dent 2019;5(2):42-5. 